\title{
Locally metastatic mucinous rectal adenocarcinoma: Imaging diagnosis with DW-MRI in comparison with PET-CT
}

\author{
MUSTAFA BOZKURT $^{1}$, KEREM OKUTUR ${ }^{1}$, KÜBRA AYDIN $^{1}$, ESAT NAMAL ${ }^{1}$, \\ AKIN ÖZTÜRK $^{1}, \mathrm{CEM} \mathrm{BALCI}^{2}$ and GÖKHAN DEMIR ${ }^{1}$ \\ Departments of ${ }^{1}$ Medical Oncology, and ${ }^{2}$ Radiology, Istanbul Bilim University School of Medicine, Istanbul, Turkey
}

Received November 22, 2011; Accepted February 29, 2012

DOI: $10.3892 / 01.2012 .663$

\begin{abstract}
A 56-year-old male patient with locally advanced mucinous rectal cancer underwent neoadjuvant chemoradiotherapy. Follow-up imaging with positron emission tomography-computed tomography (PET-CT) revealed a local response to chemoradiotherapy, whereas diffusionweighted magnetic resonance imaging (DW-MRI) showed newly presented sacral bone metastasis. Histopathologically confirmed bone metastasis and the local tumor were surgically removed. Repeat DW-MRI revealed tumor recurrence in the sacral excision zone eight months after surgery, which was reconfirmed by histopathology. This case shows the superior imaging ability of DW-MRI in the diagnosis of mucinous tumors in comparison to PET-CT.
\end{abstract}

\section{Introduction}

Diffusion-weighted magnetic resonance imaging (DW-MRI) has increasingly been used for the detection of tumors and their recurrence following surgical resection and/or chemoradiation on follow-up examinations. The performance of DW-MRI in oncological imaging has been investigated. Recent comparisons with positron emission tomography (PET) indicate a similar or superior diagnostic ability of DW-MRI in the detection and differentiation between malignant and benign processes (1-3). DW-MRI is particularly superior to PET in the distant staging of prostate cancer and the detection of mucinous tumors $(4,5)$. We report a case of mucinous adenocarcinoma of the rectum with PET-negative sacral bone metastasis and its recurrence, which was demonstrated on DW-MRI following surgical and adjuvant treatment. The study was approved by the ethics committee of the hospital and the patient consented to the publication of his case.

Correspondence to: Dr Mustafa Bozkurt, Department of Medical Oncology, Istanbul Bilim University School of Medicine, Avrupa Hastanesi, Şişhane Kampüsü (Onkoloji Merkezi), Bedrettin M. Bedii Gorbon Sokak No:1 Şişhane-Beyoğlu, Istanbul, Turkey

E-mail: bozkurtmster@gmail.com

Key words: mucinous, colon cancer, positron emission tomography, diffusion-weighted magnetic resonance imaging

\section{Case report}

A 56-year-old male patient was referred to the Department of Medical Oncology with a four-month history of perianal pain and fecal incontinence. He underwent colonoscopy and a mass lesion in the rectum was detected that started $4 \mathrm{~cm}$ above the anal verge and had a $10-\mathrm{cm}$ cranial extension. Computed tomography (CT) of the chest, abdomen and pelvis for the initial staging revealed tumor invasion of the seminal vesicles and prostate gland with enlarged iliac lymph nodes (Fig. 1). The chest CT was normal, revealing a TNM stage of IIIB; cT4N1M0. The patient's serum blood tumor markers were 7.42 and 10.39 for carcinoembryonic antigen and cancer antigen (CA) 19-9, respectively. ECOG (Eastern Cooperative Oncology Group) performance was 1 . The patient was scheduled for neoadjuvant chemoradiotherapy with 5-fluorouracil $225 \mathrm{mgr} / \mathrm{m}^{2}, 180 \mathrm{cGy} /$ day, 5 days per week for five weeks to facilitate a sphincter-sparing procedure and to decrease the locoregional recurrence rate. Following completion of the chemoradiotherapy regimen, the patient underwent pelvic MRI to determine the locoregional staging and PET-CT for the distant staging. Pelvic MRI was performed with DW-MRI using b values of 0,400 and $800 \mathrm{sec} / \mathrm{mm}^{2}$. T1- and T2-weighted conventional imaging sequences with and without intravenous contrast were also obtained.

PET-CT revealed a localized tumor in the rectum with increased standardized uptake values (SUV) (Fig. 2A). MRI showed a rectal mass adjacent to the prostate capsule without seminal vesicle and/or prostatic involvement (Fig. 2B). DW-MRI revealed a sacral mass in the left half of the bone with diffusion restriction (Fig. 2D). There was no 18F-fluorodeoxyglucose (FDG) uptake in the sacrum on PET-CT images (Fig. 2C). No bone destruction was appreciable on the PET-CT images. A CT-guided bone biopsy was performed and material sampled from the sacrum was found to be positive for mucinous adenocarcinoma. The patient underwent abdominoperineal resection of the rectal tumor that also included removal of the prostate and seminal vesicles with hemisacral excision. The surgery ended with a mesh implantation to restrict bowel motion into the pelvic cavity, to prevent intestinal side effects in case of radiotherapy for locoregional recurrence. Surgical material from the rectum was consistent with mucinous adenocarcinoma and the same as the sacral bone material. The surgical margins were all negative and there was no evidence of contiguous 


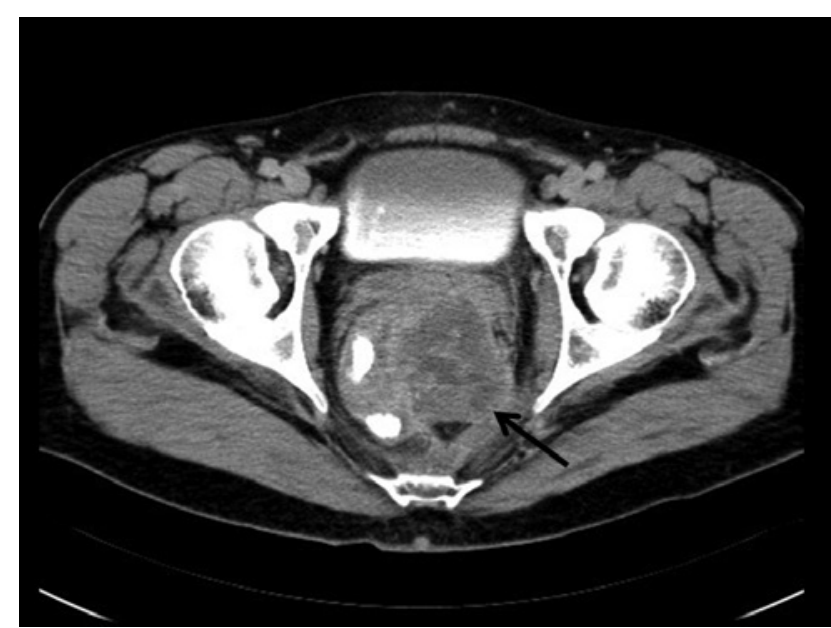

Figure 1. Axial computed tomography image from the initial presentation shows a tumor in the rectum, invading the prostate gland (arrow).

tumor invasion of the sacrum. The patient received five courses of FOLFOX-4 as adjuvant chemotherapy. Eight months subsequent to the pelvic surgery, a follow-up MRI with DW-MRI was performed, which showed a recurrent mass in the sacral bone resection zone (Fig. 3). CT-guided biopsy confirmed the MRI diagnosis. The patient was scheduled for cyber-knife treatment of the sacral mass.

\section{Discussion}

Imaging is the most important diagnostic tool for staging and monitoring therapy response in patients with colorectal cancer. MRI has been used for the locoregional staging of the tumor, whereas positron emission tomography (PET) with FDG has become the modality of choice for the initial staging and monitoring therapy response (6-9). The majority of malignant tumors have a higher rate of glucose metabolism than normal tissues. However, variations exist in the glucose metabolic rate, depending on the histological type and the aggressiveness of the tumor. Moderate and slow-growing malignant lesions exhibit a lower glucose metabolic rate than aggressive rapidly growing tumors $(8,10)$. Awareness of the potential limitations of FDG PET with particular histological subtypes is essential for the proper application of this technique. Mucinous adenocarcinoma is commonly localized in the gastrointestinal tract. Mucins are high-molecular-weight glycoproteins and their presence in colorectal tumors is associated with a poor prognosis, with the exception of colorectal cancer, for which
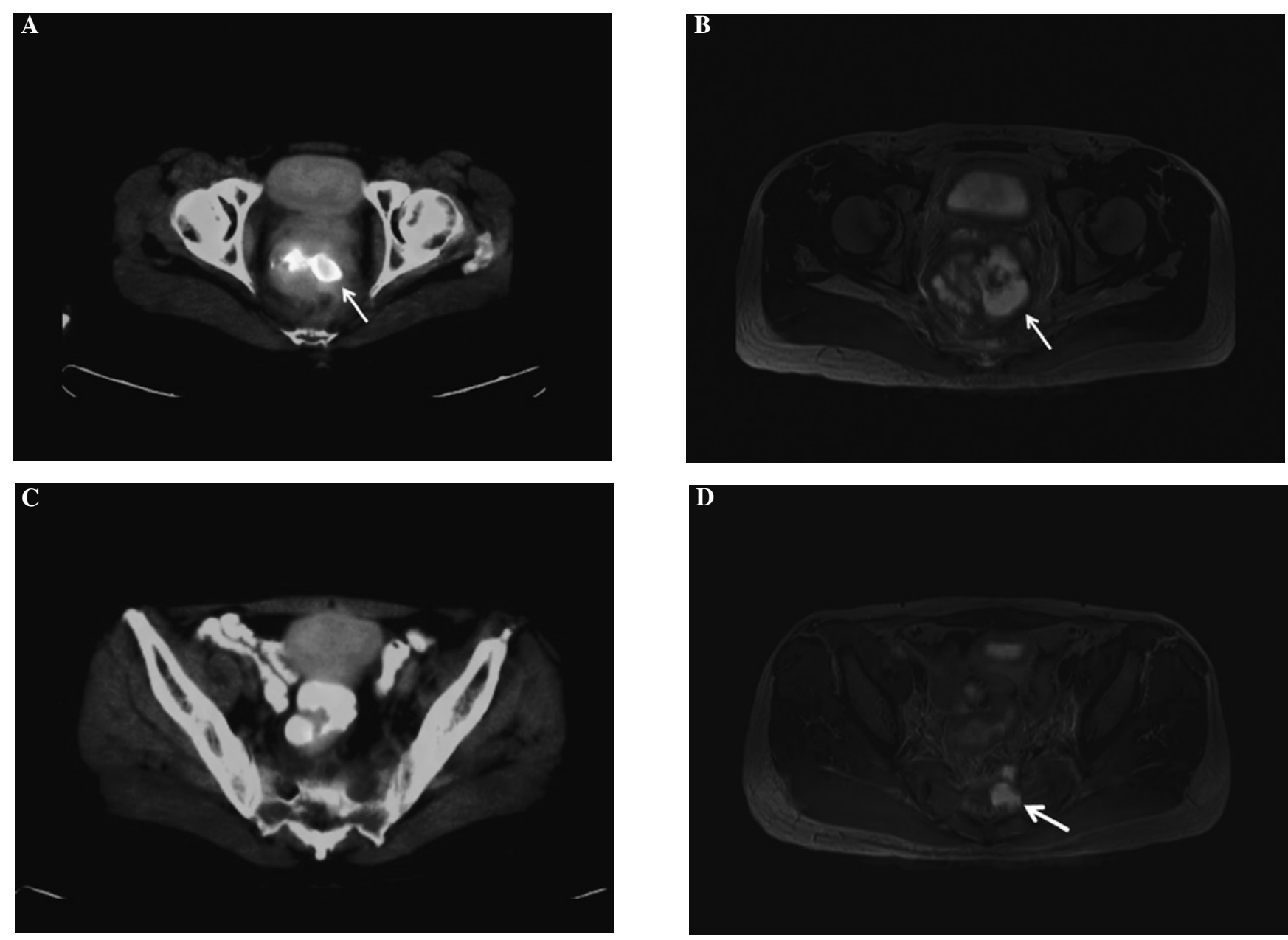

Figure 2. Neoadjuvant chemoradiotherapy reveals reduced tumor size in the rectum on (A) positron emission tomography-computed tomography (PET-CT) and (B) diffusion-weighted magnetic resonance imaging (DW-MRI). (C) No FDG uptake was observed in the sacrum on PET-CT images, whereas (D) DW-MRI shows a mass in the sacrum. 


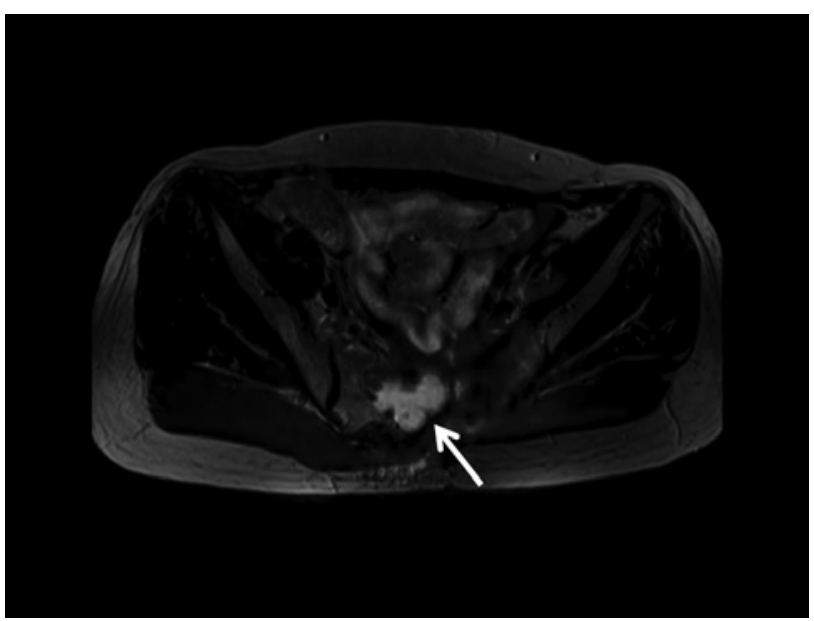

Figure 3. Eight months after surgical removal of the sacral mass, a recurrent tumor is evident on diffusion-weighted magnetic resonance imaging.

clinical studies have shown that the presence of mucin is associated with a lower rate of survival $(11,12)$. FDG uptake has been correlated with the number of viable tumor cells as well as the grade and the differentiation of certain tumors. Findings of a recent report showed that mucin exhibits a lower peak SUV of FDG than tumors without mucin content (12). Lower levels or absence of FDG uptake in mucinous tumors may be explained by the reduced number of tumor cells, which are replaced by mucin in the microenvironment, resulting in a decreased cellular density of the tumor $(12,13)$.

DW-MRI enables the visualization of Brownian molecular motion, i.e, it reflects the motion of water molecules in the extracellular, intracellular and intravascular spaces. DW-MRI qualitatively and quantitatively assesses tissue cellularity and the integrity of cell membranes, since the degree of restriction to water diffusion in biological tissue inversely correlates with the tissue cellularity and integrity of the cell membranes. Restricted diffusion is observed in tissue with high cellular density such as tumors, leading to tortuosity of the extracellular space and higher density of hydrophobic cellular membranes, whereas in cystic or necrotic tissues the apparent diffusion of water protons is relatively less limited. Apparent diffusion coefficient (ADC) values determine the ability of the water protons to diffuse. Moreover, these values are expected to be lower in viable tumors $(1,4)$. Mucinous tumors reveal higher ADC values than tumors without mucin content. However, the overall ADC values and visual perception of the mucinous tumor are distinguishable from cystic or necrotic tissues with high ADC values (14). This may explain the detection of the viable tumor in the sacrum by DW-MRI that had a low SUV on PET.

The SUV values decrease from normal to imperceptible values as early as three months following chemoradiotherapy in colorectal cancer patients, although a certain degree of decrease is expected earlier (9). In our case, the PET imaging was performed five weeks after initiation of chemoradiotherapy and was positive in the local tumor without evidence of FDG uptake in sacrum. The absence of baseline PET prior to chemoradiotherapy limited the evaluation of the treatment response in our case. Histological variants of rectal adenocarcinoma, including signet cell, mucinous, medullary, adenosquamous, undifferentiated, spindle cell, clear cell, hepatoid and oncocytic, have different clinical outcomes. These variants are almost always associated with a poor prognosis compared to adenocarcinoma of the same disease stage. Mucinous adenocarcinoma is more aggressive than usual non-variant adenocarcinomas. It is characterized as affecting younger patients and having a high frequency of lymph node metastases, local recurrence and advanced stage at presentation. Mucinous adenocarcinoma has been reported to show poor downstaging following chemoradiotherapy in comparison with that of rectal adenocarcinoma, thus surgical management is always preferred in locally advanced disease $(11,13)$.

In conclusion, we described a case of locally advanced rectal mucinous adenocarcinoma with sacral bone metastasis. PET-CT and DW-MRI revealed a rectal tumor, following neoadjuvant chemoradiotherapy. DW-MRI demonstrated the presence of sacral involvement despite physiological FDG uptake on PET in the same location. The recurrent tumor in the excised sacrum was detectable on the repeat DW-MRI eight months after surgery.

\section{References}

1. Koyama T, Tamai K and Togashi K: Current status of body MR imaging: fast MR imaging and diffusion-weighted imaging. Int J Clin Oncol 11: 278-285, 2006.

2. Kwee TC, Takahara T, Ochiai R, et al: Complementary roles of whole-body diffusion-weighted MRI and 18F-FDG PET: the state of the art and potential applications. J Nucl Med 51: 1549-1558, 2010.

3. Watanabe T: Chemoradiotherapy and adjuvant chemotherapy for rectal cancer. Int J Clin Oncol 13: 488-497, 2008.

4. Eschmann SM, Pfannenberg AC, Rieger A, et al: Comparison of $11 \mathrm{C}$ choline- PET/CT and whole body-MRI for staging of prostate cancer. Nuklearmedizin 46: 161-168, 2007.

5. Kim SH, Lee JM,Hong SH, et al: Locally advanced rectal cancer: added value of diffusion-weighted MR imaging in the evaluation of tumor response to neoadjuvant chemo- and radiation therapy. Radiology 253: 116-125, 2009.

6. Endo K, Oriuchi N, Higuchi T, et al: PET and PET/CT using $18 \mathrm{~F}-\mathrm{FDG}$ in the diagnosis and management of cancer patients. Int J Clin Oncol 11: 286-296, 2006.

7. Dresen RC, Beets GL, Rutten HJ, et al: Locally advanced rectal cancer: MR imaging for restaging after neoadjuvant radiation therapy with concomitant chemotherapy. Part I. Are we able to predict tumor confined to the rectal wall? Radiology 252: 71-80, 2009.

8. Mainenti PP, Iodice D, Segreto S, et al: Colorectal cancer and 18FDG-PET/CT: what about adding the $\mathrm{T}$ to the $\mathrm{N}$ parameter in loco-regional staging? World J Gastroenterol 17: 1427-1433, 2011.

9. Hur H, Kim NK, Yun M, et al: ${ }^{18}$ Fluoro-deoxy-glucose positron emission tomography in assessing tumor response to preoperative chemoradiation therapy for locally advanced rectal cancer. J Surg Oncol 103: 17-24, 2011.

10. Zhu A, Lee D and Shim H: Metabolic positron emission tomography imaging in cancer detection and therapy response. Semin Oncol 38: 55-69, 2011.

11. Connelly JH, Robey-Cafferty SS and Cleary KB: Mucinous carcinomas of the colon and rectum: analysis of 62 stage $\mathrm{B}$ and $\mathrm{C}$ lesions. Arch Pathol Lab Med 115: 1022-1025, 1991.

12. Berger KL, Nicholson SA, Dehdashti F and Siegel BA: FDG PET evaluation of mucinous neoplasms: correlation of FDG uptake with histopathologic features. Am J Roentgenol 174: 1005-1008, 2000.

13. Green JB, Timmcke AE, Mitchell WT, Hicks TC, Gathright JB Jr and Ray JE: Mucinous carcinoma-just another colon cancer? Dis Colon Rectum 36: 49-54, 1993.

14. Kim DJ, Kim JH, Lim JS, et al: Restaging of rectal cancer with MR imaging after concurrent chemotherapy and radiation therapy. Radiographics 30: 503-516, 2010. 